eher überraschend, denn die materialreichen Ausführungen der Verfasserin über die sozialwissenschaftlichen Charakteristika des Völkerrechts (S. 64 ff.) und dessen Wirksamkeitsvoraussetzungen (S. 86 ff.) legen kaum den Schluß nahe, daß die Kommunikation von Normen, zumal die an Private, der ein Großteil der weiteren Ausführungen gewidmet sind (S. 144 ff.), das zentrale Problem einer "Völkerrechtssoziologie" sind. Es ist durchaus fraglich, ob die Bedeutung der Normkommunikation nicht auch in der allgemeinen rechtssoziologischen Effektivitätsforschung überschätzt wird, das Völkerrecht erscheint jedenfalls als Musterbeispiel eines Rechtsbereiches, in dem es viel weniger auf die Normkenntnis der beteiligten Akteure als vielmehr auf ihre Fähigkeit und Bereitschaft, die Normen zu befolgen, und - noch grundlegender - auf ihre Einigkeit über den Inhalt der Normen ankommt.

Das soll nicht heißen, daß Völkerrechtskenntnisse der breiten Offentlichkeit wegen einer möglichen Rückkoppelung auf das Verhalten staatlicher Akteure völlig irrelevant sind, und die empirische Untersuchungen der Verfasserin zur Ubermittlung völkerrechtlicher Kenntnisse im schulischen und außerschulischen Bildungsbereich (S. 153 ff., mit dem zu erwartenden Negativbefund) sind zweifellos interessant. Nur erscheinen diese Randthemen als wenig geeignete Beispiele für die Notwendigkeit und Fruchtbarkeit einer Verbindung von Völkerrecht und Rechtssoziologie, die die Verfasserin so überzeugend vertritt.

Trotz dieser Kritik bleibt der Wert (und die Pionierleistung) des Buches mit seiner breiten Bestandsaufnahme der Beziehungspunkte zwischen Völkerrecht und Rechtssoziologie (wie auch der Forschungsdefizite) unbestreitbar.

Brun-Otto Bryde

\author{
W. Poeggel R. Meißner Ch. Poeggel \\ Staatennachfolge in Verträge \\ Staatsvertrag der Deutschen Demokratischen Republik (Ost-)Berlin 1980, 211 S.
}

Das Recht der Staatennachfolge, eines der schwierigsten und umstrittensten Gebiete des Völkerrechts, gehörte schon seit 1949 zu den Kodifikationsvorhaben der Völkerrechtskommission der Vereinten Nationen (ILC). Nach 16-jähriger Arbeit ist es 1978 gelunden, mit der Unterzeichnung der "Wiener Konvention über das Recht der Staatennachfolge in Verträge" (WKSV) ein erstes Teilgebiet der beabsichtigten Kodifikation abzuschließen. Die Normierung der Nachfolge in Rechte und Pflichten aus anderen Quellen als Verträgen ist dagegen noch nicht vollendet, während die Nachfolge in die Mitgliedschaft internationaler Organisationen vorläufig von einer Behandlung durch die ILC ausgespart worden ist. Da die Konvention über die Nachfolge in Verträge jedoch nach ihrem Art. 49 erst dann in Kraft treten kann, wenn mindestens 15 Staaten die Ratifikation oder den Beitritt erklärt haben, bleibt abzuwarten, ob ihr nicht dasselbe (zumindest vorläufige) Schicksal zuteil wird wie der Wiener Vertragskonvention von 1969, die bis 
zum heutigen Tage nicht in Kraft getreten ist. Ein durch späteres Inkrafttreten eventuell auftretender Nachteil wird allerdings mit Hilfe der in Art 7 WKSV vorgesehenen Rückwirkungsklausel vermieden.

Unter Berücksichtigung der WKSV versuchen die Autoren zunächst, den tatsächlichen Stand des Völkerrechts auf dem Gebiet der Staatennachfolge in Verträge zu erfassen und sich mit der (westdeutschen) bürgerlichen Völkerrechtsdoktrin auseinanderzusetzen. Sie legen aber trotz dieses Anspruchs keine umfassende Monographie vor, sondern beschränken sich auf einige wesentliche Probleme. Die angekündigte Auseinandersetzung hat jedoch einen sehr fragmentarischen Charakter und stützt sich nicht einmal durchgehend auf die neueste Literatur. Auch wird der Auswertung und Untersuchung der Staatenpraxis nur in geringem Umfang Raum gegeben.

Nach heutigem universellen Völkerrecht sind allein Staatenwechsel und Gebietsveränderungen rechtmäßig und werden vom Institut der Staatennachfolge erfaßt, die in Ubereinstimmung mit dem Selbstbestimmungsrecht der Völker sowie dem Interventions-, dem Gewalt- und dem Annexionsverbot stehen. Diesen auch in der Präambel der WKSV zum Ausdruck gekommenen zentralen Grundsatz heben die Autoren zu Recht hervor. Dennoch erklären sie das aus dem Selbstbestimmungsrecht folgende Recht zur Separation einzig in kapitalistischen Vielvölkerstaaten oder in Entwicklungsländern für anwendbar, obgleich die WKSV in Art 34 keine Anhaltspunkte für diese unhaltbare Auffassung bietet. Aus der Sicht des Selbstbestimmungsrechts und des Annexionsverbotes erscheint es auch als reichlich nebulös, die Gebietsveränderungen nach dem Zweiten Weltkrieg als schwerwiegende Sanktionen gegen Aggressorstaaten zu umschreiben.

Im Kapitel "Zerfall von Staaten" wird die Deutschlandfrage angeschnitten und die These vom Untergang des Deutschen Reiches im Jahre 1945 wiederholt. Die Autoren sehen in der Erklärung der Siegermächte in Anbetracht der Niederlage Deutschlands vom 5. 6. $1945^{1}$ einen Beleg für deren Absicht, den deutschen Staat zu zerschlagen. Die Erklärung läßt diese Auslegung nicht zu. Die Ubernahme der Regierungsgewalt durch Besatzungsmächte führt zudem noch nicht den Untergang eines Staates herbei, da das Völkerrecht der Staatenkontinuität zuneigt und im Jahre 1945 noch keineswegs absehbar war, daß einmal zwei deutsche Staaten entstehen würden. Der deutsche Nachfolgefall ist nach Meinung der Autoren nicht in die vorhandenen Typen der Staatennachfolge einzuordnen, obwohl sie beide deutschen Staaten als Nachfolgestaaten des Deutschen Reiches betrachten.

Keinen Eingang in die WKSV hat die von der sozialistischen Völkerrechtslehre - wenn auch nicht einhellig - vertretene Ansicht gefunden, daß eine sozialistische Revolution eine Veränderung des Staatstyps zur Folge habe und damit zu einer Staatennachfolge führe, nicht bloß zu einer Regierungsnachfolge. Das bedauern die Autoren zwar, halten es aber anscheinend nicht für so schwerwiegend, denn "die sozialistischen Staaten haben je nach spezieller Interessenlage und unter Berücksichtigung des internationalen Kräfte-

1 United Nations Treaties Series, Bd. 230, S. 190. 
verhältnisses in ihrem praktischen Verhalten zum Teil von der Staatennachfolge als auch von der Regierungsnachfolge Gebrauch gemacht".

Die WKSV regelt in Art 16, daß ein neuer unabhängiger Staat unbelastet von den Vertragspflichten seines territorialen Vorgängers seine Existenz beginnt (clean-slate-Prinzip). Damit und mit der Rückwirkungsmöglichkeit des Art 7 WKSV wurde den vielen neuen Staaten der Dritten Welt eine erhebliche rechtliche Gestaltungsmöglichkeit ihrer eigenen Nachfolgefälle eingeräumt. Andererseits ist nach der Meinung der Autoren, die der überwiegenden Ansicht der Völkerrechtslehre entspricht, jeder Nachfolger an die im Zeitpunkt seiner Entstehung bereits vorhandenen zwingenden Prinzipien (ius cogens) und alle anderen allgemein anerkannten Normen des Völkerrechts gebunden. Eine derartige Bindung stellt aber ein grundsätzliches völkerrechtstheoretisches Problem dar, sind doch diese Regeln "res inter alios acta" für neue Staaten. Das gilt um so mehr als gerade die sozialistische Völkerrechtstheorie nur eine Selbstbindung der Staaten anerkennt $^{2}$. Diese Problematik läßt das Buch ungeklärt, obwohl ihre zentrale Bedeutung für das Recht der Staatennachfolge keineswegs verkannt wird.

Im zweiten Teil der Arbeit gehen die Autoren näher auf die einzelnen Abschnitte der WKSV ein. Dabei berichten sie über viele Einzelheiten und Abstimmungsergebnisse. Es fällt allerdings nicht immer leicht, hierin einen Nutzen zu erkennen.

Dem Buch ist eine schon seit geraumer Zeit sich abzeichnende Tendenz eigen, auf Polemik gegen die Bundesrepublik Deutschland weitgehend zu verzichten und die Völkerrechtsdiskussion zu versachlichen. Deutlich wird auch, daß selbst die offizielle sozialistische Völkerrechtslehre keineswegs einstimmig ist; die Autoren lehnen nämlich die von einigen polnischen und sowjetischen Völkerrechtlern vertretene These von der Staatenidentität bei einer sozialistischen Revolution ab, nachdem sie diese abweichenden Ansichten haben zu Wort kommen lassen. Nimmt man das 1973 erschienene Völkerrechtslehrbuch der Akademie für Staats- und Rechtswissenschaft zur Hand, so wird dieser Wandel sichtbar.

Hans-Heinrich Nöll

\section{Werner J. Feld}

\section{Multinational Corporations and U. N. Politics - The Quest for Codes of Conduct}

Pergamon Press, New York, Oxford, Toronto, Sydney, Frankfurt, Paris 1980, X, 173 S., $\$ 25$

Das Buch beschäftigt sich nach einem knappen, aber anschaulichen einleitenden Aufriß der politischen und ökonomischen Problematik der multinationalen Unternehmen mit den UN-Arbeiten an Codes of Conduct. Den Schwerpunkt bilden dabei die im Rahmen des Wirtschafts- und Sozialrats geführten Diskussionen um einen generellen Code of Conduct, aber auch die Arbeiten von UNCTAD und ILO an Spezial-Kodizes (Wettbe-

2 Vgl. Tunkin, Völkerrecht der Gegenwart, 1963, S. 78; Schulz/Hugler in Völkerrecht, 1973, S. 260 ff. 